\title{
Analysis of Medical Markets and Use of Mechanism Design Physician-Patient Interactions and Role of Organizations
}

\author{
Christine Claude Huttin ${ }^{1,2}$ \\ ${ }^{1}$ School of Management, University Aix Marseille, Marseille, Aix en Provence, France \\ ${ }^{2}$ ENDEP US Research, Inc., Cambridge, Massachusetts, USA
}

Email address:

chxyinfo@endepusresearchinc.com, christine.huttin@univ-amu.fr

To cite this article:

Christine Claude Huttin. Analysis of Medical Markets and Use of Mechanism Design Physician-Patient Interactions and Role of Organizations. Science, Technology \& Public Policy. Vol. 5, No. 1, 2021, pp. 29-39. doi: 10.11648/j.stpp.20210501.13

Received: December 11, 2020; Accepted: January 8, 2021; Published: March 26, 2021

\begin{abstract}
This paper has been written after a seminar at the Soton business school (University of Southampton), discussing how market design mechanism can be useful for some medical markets, especially in combination with the reversed algorithm, called reversed conjoint model on physicians' choices (Huttin, 2014, 2017); this first paper provides a description of two cases, one at the micro level of physician and patient interaction and a second one to integrate the organizational level of primary care groups. The first case is a micro representation of a physician-patient interaction, which can be expanded to integrate various types of heterogeneity of demand for care. The second case introduces the organizational level of primary care, with the example of the fundholding system; it leads to opt for a cooperative approach and to integrate variations between over spenders and lower spenders' physicians. This paper uses the formalization of a game approach and proposes a stream of research for policy design in decision tools for financing reforms in health care. It explores how market failures in health care can be addressed with engineering economics and implementation of rules for different types of interactions in medical markets. Moreover, the limits to address the sources of heterogeneity between practices with tools such as yardstick competition may be addressed with the fast digitalization of the health system.
\end{abstract}

Keywords: Medical Markets, Reversed Mechanism, Physician, Primary Care, Market Design

\section{Introduction}

The current research stream on physicians 'choice models under the collaboration between Professor Christine Huttin and Professor Jerry Hausman aims to better understand the impact of physicians' choices, either prognostic, diagnostic or treatment choices (especially drug choices) when patients are covered under public versus private insurance schemes or need to decide to switch from private plans to the public plan, Federal Medicare coverage. This research question is investigated with new types of mixed logit models to analyze the impact of heterogeneity of physicians' choices and patients' characteristics, for various decisions with and without independence of irrelevant alternatives among the choice sets.

It leads to improve statistical estimation of predictive expenditure models from conventional logit or probit forms by using random preference components for prices of medical services, proxies for various public and private insurance plans in addition to fixed characteristics associated with patient profiles such as age sex and risk factors or comorbidities (e.g., obesity) and new specification tests on Independent of Irrelevant Alternatives assumptions (IAA). These series of models move the development of the economic models away from the type of decision models used in medical schools, mainly called the step models, usually representing the likelihood of providing a treatment and the patterns of utilization and expenditures of various medical services (e.g. drug utilization).

The mixed logit model development, especially with random preference variables, aims to integrate the price of goods and services and therefore supply and demand interactions in physicians' choices. However, they are still limited to integrate all the actors that constrain and influence the markets.

Even if a large literature exists especially on induced demand and induced supply [1], it does not provide an indepth analysis of the stakeholders behind. Moreover, many 
hidden interconnections are unrevealed, therefore it is also important to explore additional approaches to ensure the stability of markets and control the impact of large uncertainties, especially on the demand side. This is particularly relevant in the middle of the Covid 19 public health pandemics, in order to ensure adjustments of supply and demand on medical markets and avoid major and global disruptions.

The scope of this paper is to formalize the main interactions of the medical markets with an approach from game theory, in particular the one called market design approach, in order to discuss how reversed algorithms, especially algorithmic pricing (including the reversed conjoint model) can be of use in such markets; it also starts a more comprehensive identification of players and their interactions. Contributions of game theory approaches are very useful, since they usually provide a state of play with different players to analyze how they negotiate, cooperate and compete in various forms of games, according to types of markets; a review of main types of game modeling approaches for the medical market would be very useful but is beyond the scope of this paper. This paper provides an analysis of two cases, one to describe the micro interaction at the individual level between a physician and his patient; a second one to integrate the organizational level and interactions between groups of physicians within and between medical practices. It uses previous research materials and a selection of existing publications on medical market analysis from economists using game theory.

\section{Background on Game Theorical Approach for the Medical Markets}

Game theory has been very used in the field of Industrial organization to represent the interactions between players in various types of markets and industries; before we discuss the interest to complement the current streams of research, it is useful to summarize the main categories, especially what is relevant for this analysis of health care systems, especially primary care.

According to Christian and Griffith, the field of game theory can be classified in two main approaches [2]:

1. The conventional game approach, which analyzes "what behavior emerges, given a set of rules".

In this approach players are interdependent, and the game helps to analyze interactions between strategic choices of each player: for instance, a strategic choice of player A will influence the strategic choices of the other player; a game theorist will provide what is called matrices of pay offs.

2. The reversed game theory approach, which deals with "what rules will give behaviors we want to see".

The "reversed game theory" is also called Market Design and is a field in game theory studying solution concepts for a class of private information games. In this approach, the game designer will revise the sets of rules to obtain the behaviors he wants from the players (e.g., with the design of incentive mechanisms).

The use of game theory in both cases, requires the identification of players in the game and possible need for a third party, such as regulators. This is especially true of the health systems, when they are heavily funded on taxes and public schemes, in addition to private payers. There are many developments in the field of game theory, especially applied microeconomics tools; however, this classification is relevant in this paper, since it also introduces the concept of a reversed approach, especially used for market design. The design of mechanisms for resource allocation in analysis of economic system originated with the work from Hurwicz [3, 4], in continuation of the mathematical approach of games by Van Neuman and Morgenstern [5]. Among main contributors, we can cite also cite Maskin, Myerson, Harsanyi, Roth [6-8]. In their recent review of the evolution of the field of game theory, Roth and Wilson [9] also suggest that market design can be useful for market analysis with a bigger economic environment, to incorporate especially actors and stakeholders, who cannot stand the functioning of market economics. This applies quite well to medical markets, especially in socialized health systems, strongly driven by ideology (e.g. to favor equity in access to care and address inequalities in health). Such an approach has been applied in health care markets for instance on kidney organ exchanges and compatibility incentives $[10,11]$, design of incentive mechanisms for cost reduction in home health care, for mobile crowding sensing [12] or for social awareness of services.

For interested readers, other useful development in the game theory literature, relevant for life science and health care sectors can be used such as "Evolutionary game theory" (see the recent reviews from Roth and Wilson [9] or Glaeser [13].

In this paper, the analysis of the medical market with a game approach focuses on interactions between physicians and patients, with two cases: the first case of a noncooperative game and a second case of a cooperative game. Additional research is also under progress to map relevant additional players for the pharmaceutical system, including public and private players of the R\&D ecosystem. However, it will be used for further development in the research stream on engineering approaches in life science and health care by Huttin [14], since physicians are at the interaction of supply and demand.

Beyond the previous classification, it is also worth providing a definition of algorithmic mechanism design, since the objective is to consider this approach to implement algorithms such as the reversed conjoint design used for cost sensitivity simulators in health systems, using computing infrastructures and IT architecture. Moreover, it needs to be combined with the main areas for algorithmic development in life science and healthcare" [14], leading to generation of decision tools for adjustment of supply and demand, implemented with organizational changes and in learning health systems. 
The first definition of algorithmic mechanism design comes from a paper by Nisan and Ronen [15], combining economic game theory and computer science. It mainly differs from economic mechanism design by also considering computational constraints. According to these authors "it combines ideas from economic game theory such as utility maximization, rationality and Nash equilibrium" with concepts from computer science such as "complexity and algorithm design from discrete mathematics and theoretical computer science". These definitions help to position the first series of cases presented in the next section, on medical markets, and may be useful to implement "reversed algorithms" on some parts of medical systems.

\section{First Game Case: The Microlevel of the Interaction Physician-Patient}

This interaction is usually the first important characteristic of the medical market. A physician decides with more or less involvement of the patient, according to types of diseases; physicians'choices are clinical strategies for different pathways, including decisions such as initialization decisions (option to wait to initialize a treatment or not), decision to order tests or not. Choices among alternatives for different options vary according to the current stage of knowledge in medical sciences, but also with existing limitations of access to coverage and listing of procedures and products, in the health system where they practice.

The example on the physician-patient interaction at the individual level, with a game theoretic approach is described by economists Su-Sheng et ales [16], who addresses the cost and quality trade-off, addressing the asymmetry of information, at a micro level, between physicians and patients. They use the formalization of a conventional non cooperative game, with the use of a Nash Equilibrium model. The game approach is formalized in this way:

\subsection{Form of the Game Between Physicians and Patients}

The form of a game between the physician and the patient, who are the two players in that case, provides a basic understanding of a game approach for design of medical markets.

Medical markets are characterized by the role of physicians at the interaction of supply and demand, who decides to a large extent for patients, this game approach represent the Micro decision, at individual level, with a starting point, that can then include different types of variations according to types of physicians, practice groups, and types of decision making processes (e.g. categories from paternalistic to shared decision making approach [17]) and various forms of information processing and sharing between the two players.

Contrary to the psychological models such as the Brunswik lens model and other types of clinical judgment analysis approaches used in my previous work on economics and medical decision making [1], the formalization does not mention if, how or when some evidence is used during the decision-making process.

\subsection{Strategy Sets}

In this formalization for a medical market, the cost-quality trade-off is simplified to represent the patient's strategy and the physician's strategy:

"the Patient's strategy is his cost function"

"the Physician's strategy is the quality function of the medical service"

This simple model does not include at this stage the patient satisfaction for his needs in term of quality (which is dominant in some western states) beyond the role of only medical services (including when to access them). In the same way, the service quality function does not integrate the needs to include the budget restrictions imposed by the organizations (either medical practices or hospital organizations) or business pressures with consolidations or mergers of hospitals).

The authors are coming from Asian markets and access to care and affordability seemed to be the dominant criteria, so the authors prioritize the formalization with economics and affordable strategy on the patient side, and quality on the provider side. It may anticipate the role of data models and machine learning in following steps to train the model with data from different locations and organizations of care.

My previous contributions, especially with the evaluation research group in Europe for pharmaceutical policies, also started from that individual level, but it was not limited to patients and physicians; it also included the pharmacist, since in Western health systems, it is often between patients and pharmacists, that the economic or cost issues prevail and are discussed.

The cost function and the patient-physician interaction are more or less dominant according to the health systems; if the critical decision point to discuss costs in the medical system is the physician then it makes sense to compare the formalization of a simple interaction for cost /quality described with some of the health systems where doctors 'cost reduction strategies are dominant (see comparison between France and Germany in the series of case studies for critical decision points for random utility models in pharmaceutical systems [18]).

However, for medical markets, the simple representation of the basic game model focusing on cost/quality trade off may allow future development for weighting experiments on cost and quality, that could differ between the two types of players for various reasons.

\subsection{Information Set}

The key information needed and shared in this medical market is the patient illness and its health status, there is no mention at this stage of potential perceived health status versus quality-of-life type of measures at different disease progression stages. The two players have different estimations of the illness: 
The physician processes clinical information, using the support of health care organizations ("physician's market power"'[16]; he can order tests, exams and different procedures based on his own medical knowledge, but also his experience or shared knowledge via the consensus built during various revisions of clinical guidelines. Other professionals also help more and more with additional knowledge such as geneticists for interpretation of genetic tests. Moreover, the prognostic and diagnostic space is largely dominated by the physician, since it is hard for patients to challenge this access to relevant clinical information and its interpretation.

The patient information set however can also be very deep, since the information is HIS own data (gene society). The more experienced the patient is with the disease or connected to social media vested into health, the more he also becomes very knowledgeable about his own case, and searches information especially with the help of new social media, sharing with patients with similar diseases.

This may appear a challenge for physicians, but if the relationship or the interaction (using the game model approach terminology) works well, then the scope of each information sets largely increase the likelihood to find the appropriate clinical strategies and may increase the accuracy of the main judge (the physician).

This information set is critical to assess patients'needs but is also constrained by the organization and the pace of access to the results.

The current fast digitalization of health systems may also need to be controlled in such models, the adoption of new modes of communication has direct impact on the players and their strategy; computerization of information speed up interactions and limit their cost. It may also change the original strategies of the players.

\subsection{Game Form}

This appears more an issue for the analysis of medical markets. In a game approach, players bargain usually or cooperate

However, interactions between physicians and patients are usually more seen as forms of collusion or even tacit collusion.

A physician with his ethical commitment, usually try to treat his patient and make sure he can access an affordable care. So, he may also "game" the system: for instance, he may help his patients to be exempted, if he judges that it is necessary to access care, or he may direct his patient to dispensary or other free access to care, according to his patient's affordability.

However, the non-cooperative aspect of the game model presented in this first case between physicians and patients, does not reveal these behaviors, but mainly reflects different economic objectives of both physicians and patients.

This will be further discussed in the second case of a game integrating the organizational level, in the next section. A physician also needs to secure his own practice and remuneration (especially for instance in medical or health systems, where the budget holder for services is also the practice and the prescribing budget is calculated in a total budget that also includes the physicians 'remunerations).

Moreover, within total health care budgets, the trade-off between the two budgets: pharmaceutical budget and doctors' remunerations, could modify the bargaining form of the game between the physician and the patient (for the levels of quality or appropriateness of healthcare services provided to him) since to a large extent, they are both dependent on the same budget. The game would need to be extended to include other layers of interactions on the trade-off for two economic stakeholders: the industry actor and the physicians. It would not be limited to the simple interaction of the physician and his patient.

\subsection{Functions of Pay-off}

The case described as a simple game of the physicianpatient interaction presents two pay-off functions with two estimations of the illness. The pay-off function represents:

1. "The utility for the patient: estimation of the Illness by the patient- Price"

2. "The utility of the physician: Price - estimation of illness by the physician"

This basic game form can be expanded in various ways, but it remains useful, since it concentrates on the key priority which is normally to address patients' needs with the help of medical systems.

For the patient, a pay-off, in the terminology used by game theorists, correspond to the economic concept of utility, which in the micro economic theory, is represented by series of indifference curves.

The pay-off for the physician also represents his utility, namely a physician' $s$ preferences for his patient and therefore, his treatment choice sets. These sets can be more or less limited, according to various factors: the main one is the type of medical technologies available where the physician practices, but also what he is willing to consider as choices, not only for the patient needs, but also for promoting the path towards new types of treatments.

Physicians are then classified between innovators and followers. The first group, often goes beyond the clinical guidelines, usually with patient consent (e.g. with investigational drug therapies). They also promote aggressive preventive strategies for instance, or newest treatments that might work when existing treatment on the market have limited effectiveness at least on some categories of patients. Followers are usually physicians who comply to current clinical guidelines. However, the current COVID-19 crises confirm that most doctors face various patient needs and complex cases, with limited understanding of the effects of existing therapies, and they need to adjust or experiments with treatments, accordingly.

Moreover, these two categories may become very different with the emerging machine learning techniques that again, train data models and support physicians and patients with available knowledge and understanding of how bad or severe is the stage of the disease or illness, and what can be their 
options.

The main interest of this game formalization is a microrepresentation of the physician-patient interaction, that can be expanded, to integrate various types of heterogeneity. It can help a micro economic theory of the demand for care, and different forms of linkages and aggregations of physicians and groups of patients.

The main limitations are the lack or limited knowledge and understanding of the sources of variations of patients'health status and the lack of explicit knowledge of interplays among groups of physicians (e.g. peers, different medical societies) and clinical governance issues. Moreover, such a game form may need additional development under the pressures of tech companies or other big data players, in the context of open boundaries in Science, beyond conventional boundaries of clinical systems.

\section{Second Game Case at the Level of a Health Care Organization: Budget Holding System in Primary Care}

After describing the way to formalize basic micro interactions between physicians and patients for the analysis of medical market, the second case aims to also adopt this type of formalization, with an integration of the organizational level, looking at the way drug budgets are allocated between physician groups within a larger organization of primary care groups.

\subsection{Case on Medical Group Organization, the Fundholder System}

This case was part of a research project including series of qualitative interviews of various types of medical practices, during the reform of the budget holding system in the UK [19-21]. The reform took place in various countries: the UK, Israel, New Zealand, Germany for instance, to make the physician profession accountable for the resource allocated to primary care services, within the total health budget. The materials used to formalize this game, is mainly from the UK at the time of the reform, when Primary Care Groups were accountable for drug budgets (1).

The first game was not only limited because of the micro decision between a physician and a patient but also because of the type of non-cooperative game. If the organization where the physician practices is also integrated in the formalization, then the first game would represent mainly the case of solo practices. These cases are rare now. Even when practices are small, usually peers and professions collaborate at least for consensus guidelines; so organizational forms in a health system and the power of the medical associations need to be integrated. Moreover, inside primary care groups, there is a need to represent the interplay between physicians and groups of physicians.

The fundholding system is a good example to analyze interplays between physicians. Objectives of such a system are mainly the following:
1. To foster exchanges of experiences between practices

2. To encourage practices to work more together

3. To use peer pressures to enforce quality improvement

4. To increase cooperation and avoid self-interest behaviors

5. To diffuse innovative treatments

In such an organizational form, the budget constraint is partially transferred in the system to make physicians accountable for the allocation of budgets for prescription drugs, tests and access to hospital services.

Contrary to the first game, this case led to formalize the game as a cooperative game. Since it tends to increase cooperation between practices and to avoid self-interested behaviors, in order to adjust drug budgets in fair manner between the set of practices.

Such a system was studied in a noncompetitive health system (the NHS), but it also exists in privately dominated system or competitive health systems such as the US system.

\subsection{Cost Quality Trade-off for Prescription Drugs in a Primary Care Group (PCG) and Incentives Mechanisms}

The players in this game are physicians' budget holders in different types of medical practices. In such a system, the practice groups have also a financial managerial role. The key budget issue for their board is to decide how to allocate the budget, in the future, facing practices with different levels of drug spending. A PCG faces different categories of practices: the overspenders, the underspenders or low spenders and the average spenders.

Among the "overspenders", there can be good and bad reasons for overspending. Among the good reasons, there are practices, which prescribe new innovative drugs and a higher diffusion of high-tech drugs in general. The argument is that these drugs are usually more cost effective and tend to provide evidence either on saving, especially for secondary care services or better morbidity figures for the treated conditions. However, one challenge is that the diffusion of these new innovative drugs is strongly influenced by pharmaceutical companies who have direct commercial interest in pushing their own drugs for certain therapeutic areas. It may therefore influence targets for setting higher budget allocations for certain diseases or certain type of drug treatments. Other reasons for overspending can be linked to specific health needs of the population like more severe conditions. Among other reasons for large overspending, we can also find a slower adoption of the different methods used to rationalise prescribing and limited cost awareness about the implications of different prescribing habits.

A game approach can formalize how such professionals can reach the objective of their drug budget ceiling between two groups of physicians "overspenders" and under or average" spenders inside the organization, since the group is accountable for a share of the collective public budget.

(1) The fundholding system has been reviewed in different countries, it still provides interesting lessons on cost containment policies in primary care [e.g. 22]. 


\subsection{Positions of Fundholders and Non-Fundholders}

The next section describes positions, interests and opinions of practices who opt for the fundholding system, called the fundholders, who refused the new system, called the non fundholders, and how they could operate, in order to benefit from larger budgets.

Position of a Fundholder:

Fundholders were well known to be lower spenders on drug prescribing. One reason is that they had stronger incentives to reduce cost on prescribing, to shift the saving for other parts of the budget they had also the responsibility on. Practices entering the fundholding system had incentive to raise substantially their historical budget in order to bargain for a larger budget upfront. They introduced cost saving measures within the practice such as a high rate of generic prescribing, options for not prescribing, repeating prescribing management, hiring a pharmacist to review their prescribing. They usually used a clinical governance lead, to provide recommendations on prescribing and to help the diffusion of innovative practices; it is not obvious he could generate more efficiency. In the new organisational form, to a certain extent, incentives to manage cost down are reduced in the new organisation in comparison with the previous one, since they have now to share direct responsibility for the drug budget, and cannot directly reallocate the savings on other items of their own practice. Moreover, potential budget increase for a fundholder organization seemed to have decreased from what the former increase it could get as an independent entity (e.g. increase of $+1.3 \%$ /year versus 6 to $10 \%$ the previous year). It is not surprising it was in a position to become overspender. This was confirmed in the interviews within the PCG' Chief executive. Some of the former lower spenders started to slip and the group of practices started to overspend his budget. It is interesting to understand the arguments advanced by the fundholder practice to justify his overspending. The main argument was quality and use of new drugs. This may be a very realistic argument in the context of rising prices of new drugs, technological trends in the pharmaceutical sector and a relatively low percentage of healthcare spending, as percentage of GNP. The PCG defended his position not to consider the drug budget per se but globally with other health care expenses and to propose markers of quality for prescribing instead.

However, the "good" performers had also less incentive, within the PCG, than before to save cost; at the same time it may lead to a looser behaviour in relation with costs (why a practice would get a lower budget to cover the overspending of the overspender practices in the PCG?). A good performer gets incentives to provide arguments to promote costeffective drugs and can be, to a certain extent, at the forefront for the group to promote some areas of potential cost savings for the global budget. To a certain extent, such practices within the PCG can be used for their level of experience and expertise in experimentations of cost saving exercises.

In addition, the PCG clinical lead had to control for two potential threats: the first one is to make sure that such practices are not guided by self interest in relation with the budget level and second, that the type of proposal is not too influenced by vested interests. A good example is the selection process of disease areas and types of drugs, which need to be or not to be considered as areas of cost savings. In the case study, the interviewed fundholder provided a detailed explanation of the process. In order to perform a detailed analysis of the prescribing and explore which areas could represent potential cost savings among different types of diseases, two review exercises have been performed. The first one was performed by the educational outreach and pharmacist department of a University and funded by a Health Authority; an educational branch of a pharmaceutical company performed the second one. Even if a physician believes that he can judge what could be a promotional bias versus objective information, his choice may lead to promote the use of certain types of drugs for certain diseases, to the detriment of other types of diseases and other types of drugs (e.g. respiratory drugs versus cardiovascular drugs). Within such disease management approach, physicians also face a new form of competition between companies. The promotional effort is shifted to educational branches in order to push the most cost effective drugs, not only in relation with other drugs for the same treatment; but the companies get also involved in the analysis of the prescribing of therapeutic areas where they do not have drug treatment, in order to recommend potential savings areas to make in such disease areas.

Therefore position and preferences of a practice, for the selection of drugs and diseases should be considered as a proposal in a larger selection process which could guarantee that non vested interests are leading the decision making process for the whole group. The freedom of choice of each physician and each practice, if it is preserved and maintained as a rule in the PCG, will allow a diversity of opinions and potential very useful initiatives. To a certain extent, the PCG framework can be used to provide a platform to discuss the countervailing sources of information, which contribute to different set of preferences among physicians. Even if national sources exist to guide and inform the medical profession on the best evidence for some treatment, when it comes down to a practice level, the physician still faces some trade-off choices, according to specific needs of its registered population and cannot directly transfer national or regional guidelines into his own practice (e.g. Health Technology Assessment Agencies (HTAs) recommendations, such as "Nice", or other evidence generated at national or regional level).

Position of a Non Fundholder:

A number of practices refused to opt for the fundholding system and it is important to examine the positions of a nonfundholder group in the organizational national change of the primary care sector. These Medical Practice Groups opt out for different reasons.

We can identify two types of practices among the non fundholder groups: the practices who clearly opposed the fundholding system and were determined against it; on the 
contrary the practices, willing to join, but who could not meet the conditions required (e.g. case of former groups of singlehanded practices who finally founded a collaborative arrangement in a multi-fund).

The clear political position expressed by some physicians against the fundholding system reveals another set of preferences for a certain type of organization of primary care: one argument expressed by the physicians against the fundholding system is the introduction of more fragmentation within medical care. The change would have contributed to a certain extent to lessen the cohesion of the teamwork between physicians and other professionals such as health visitors and nurses for elderly. The non-fundholder practice therefore who deliberately opted out from the fundholding system for this reason, aimed to maintain this enlarged health care team outside the practice. This opinion could therefore be very supportive of a policy option to work not only with different practices, but also to rely on larger Primary Health Care teams. Such non-fundholders' opinions remain questionable, since some fundholders would argue that they would do more teamwork since they could recruit more staffs internally.

On the budget side, this may mean that a smaller proportion of the budget is allocated then to the physicians working in the practice, contrary to the former fundholder who argued for a larger budget in order to pay for pharmacists' services or nurses' services within the scope of the practice. Therefore, it could mean that rationally, the nonfundholder would bargain for less than the former fundholder. On another hand, it had much less experience and incentives to manage the drug budget.

The divergence of physicians 'opinions on the impact of fundholding schemes on community teamwork has been assessed in the literature on the fundholding system reforms.

The requirement imposed on a non-funholder was to follow a budget scheme, which was only indicative. Therefore, the constraint on prescribing costs was much lower than for a fundholder. A non-fundholder could either be a good performer or a bad performer in term of overspending or underspending, according to his goodwill to perform so. In the context of the PCG, the budget allocation by weighted capitation (called ASTRO-PU), considering that population needs are not so different for the practice than from the nonfundholder PCG average, would not normally affect the incentives for the physicians to manage the drug budget differently. However, the non-fundholder PCG also tried to stimulate the practices, to better manage drug budgets with possible cooperation with fundholders, more experienced in the field.

Physicians in non-fundholding practices, did not have the same pressure as in the fundholding system, to become cost aware, and also searched for other solutions to manage their drug budget. A practice in a non-fundholder group could find some good solutions too, since he received bonus within the incentive scheme in place by the Health Authority (HA) and probably had quite a rational prescribing behavior from the perspective of that HA. One possible explanation could be the strong belief and attitude to care for another type of organization of primary care and a willingness to defend a certain type of care with a great sense of community. The reduction of drug spending was achieved by less sophisticated ways, than in the case of a fundholder who performs thorough analysis of prescribing patterns, with support of pharmacists based on evidence from university centers, as countervailing information from the more questionable source from pharmacists recruited by a pharmaceutical company.

The main measures used by the non-fundholders to implement some cost saving measures, were the following: generic prescribing (around $72 \%$ at the time of the study, which was above the national average (64\%)). This practice was therefore already above the target. Such a behaviour showed that even if the fundolder had more incentive to increase his generic prescribing target, the non fundholder did not have lower performance on such an indicator of drug cost minimisation. The main difference need to be examined in the more sophisticated means used for cost minimisation strategies and quality of care. The second measure used by the non-fundolder was to opt for the development of a practice formulary. The formulary covered gastrointestinal drugs, cardiovascular drugs, respiratory diseases, CNS drugs and drugs for the skin. The strategy followed is then to reach a consensus among the physicians of the practice for the cheapest drugs. The formulary is revised every three or four months, the price information (using MIMS monthly information basis, as price data). The cost minimisation strategy is therefore to select preferred drugs rather than to use evidence for cost-effective drugs, requiring a much higher level of information and knowledge on the different types of drugs and not only drug price information. To a certain extent this reveals a different type of information search, at an individual level, and therefore type of knowledge available to guide physicians' choices in the two practices. Another difference with the fundholder practice is the fact that in the first case the physician tends to rely more on external sources of information coming from experts (academic or pharmacist from a company) while in the second case, the physician tends to rely (except for the price information) on the experience of the practice ("he wants it to be based from the community experience"). An example is the physicians' experience on his asthmatic patients. The physicians performed an internal survey of 67 asthmatic patients (stabilised patients) to test the feasibility to reduce the use of inhalers or the doses of inhalers.

From this analysis of physicians' behaviors in fundholder and non-fundholder systems, we can understand the reasons for overspenders or reduced drug spenders; the ways and appropriateness of the approaches may be based on more or less elaborated scientific evidence. The types of motivation in both cases are probably quite different. In the first case, the financial incentive has been influential (confirmed by the literature); in particular physicians could reallocate the savings from the drug budget on other aspects of their practices; the second case may cover different types of 
behaviours: some physicians will argue they have a more community approach; it can however mean that they are not so keen on keeping all the technological advances.

In order to pursue useful drug management policies, the various PCGs may only need to maintain physicians' motivations in both clinical settings, but also to ensure that the strategies used are the best and that the two types of practices may cooperate and share some experience. An important difference between the two types of practices is the management style: the fundholder has a board with a physician as a board member, while the non-fundholder is not represented on the board and is sometimes sending a practice manager to the council meeting of the PCG rather than physicians, showing a relative lack of interest, motivation or recognition by non-physicians, of its potential role for the management of the PCG. This may reflect a different power base of the medical profession inside each type of organizations. The non-fundholer may require external credibility (e.g. by using comparative performance tables, called at the time of the study "Beacon practices") (The Beacon practices are usually best practices approved across UK regions for certain expertise in different practice areas.) It is probably a valid argument to say that scientific evidence usually requires sound statistical validity and try to benefit from serious meta-analyses of existing scientific studies, performed by specialised experts involved in building these evidence. However, recent research on primary care tends to show that "evidence in medical practice is often represented by bodies of contestable and debatable data, and diffusions of innovation at the level of practices is dependent on individual conceptions of credible evidence" [23]. The bottom up approach may be questionable but a strong community experience, tied up to the needs of the population can also bring additional important elements in a selection process of drugs and prioritisation of disease areas, mainly based on the state of scientific evidence available at a certain point in time. The pay-off matrix on physicians' cost reduction strategies can be represented by the following table. Contrary to the first case, in order to optimize the budget allocation, forms of cooperation in the 4 categories are searched by a third party (the government).

Table 1. The Pay-off Matrix between groups of physicians.

\begin{tabular}{lcc} 
& Fundholder Overspender & Fundholder Underspender \\
\hline Non fundholder Overspender & \\
Non fundholder Underspender & \\
\hline
\end{tabular}

This second case represents different physicians' behaviors and sets of preferences, for managing the prescribing budget constraint. We could identify various sources of variations between the types of practices. In particular, some non fundholders or multi-funds may use less sophisticated means and had less incentives to revise their prescribing allocation than fundholders; on the contrary, fundholders were used to bid for more money and may find unfair to be penalised as they may have already been more efficient in the management of the drug budget, this may be an incentive for overspending.

An additional case, not reviewed in this paper is the group of dispensing practices, they represent a limited number of physicians in the country, but the case also reveals a total different set of issues: physicians have purchasing habits that may be inflationary but possibly useful to the PCG to understand drug prices on the market, it potentially raises individual physicians' negotiator skills.

This analysis reveals different logics and sources of experience of drug cost minimisation, which could be usefully combined. For instance, the various experiences in information search from external sources to community experience could provide countervailing information in order to find fair solutions between the different needs of the populations and the promotion of scientific evidence on only a share of the drug market. A selection process of priority setting for diseases and drug areas at the PCG level will need to control for the potential effects of the competition between various educational policies of pharmaceutical companies; therefore the use of comparative information from other
PCGs may be, at this level, a useful complement.

Forms of co-operation between different types of practices are clearly identified: using more sophisticated cost effectiveness studies than cost minimisation studies to make judgements, exchanges on procedures or more use of repeat prescribing, combination of external versus internal sources of information and forms of diffusion of scientific evidence.

The discussion of this case on physicians' organizations, confirms that under the public budgeting rule, reducing the budget often leads to lose or reduce the total budget of the organization, negotiated every year. Good performers need to keep incentives to continue to perform. They need to know how much they can keep, if they have good performance, under what kind of incentive mechanisms and for what purposes: conferences, better equipment, more enrollees?, or how their own remuneration will be affected? (contracts are usually discussed at two levels, the practice level or annually between the medical profession and the government). This kind of governance and incentive schemes do not seem right and does not automatically lead to improve quality and access to more cost-effective pharmacotherapies with a fair and equitable manner.

\section{Conclusion}

The paper reviews the interests of some types of game formalization for the analysis of medical markets and shows that additional mechanisms with a possible market design approach could be beneficial for such analysis. It could help to implement matching algorithms in addition to the research 
stream of the original reversed conjoint models, designed as decision support tools for key decision makers in health systems. So far, the ways to implement such reversed or matching algorithms system was first designed for a restrictive context of practices 'offices, in a medical system (Appendix 2); however, it needs to take into account the complex interactions, described in the second case, in order to manage the budget constraint and deal with innovative treatments, with different sources of information (companies, other educational branches) and different levels of evidence. Research in progress will also provide more comprehensive list of players, especially from the R\&D environment. As centers of excellence are usually also used by industry, an additional mapping of interactions in R\&D infrastructures and the way competitive evidence are raised and compared at the end on the marketplace is critical. Physicians entering this kind of countervailing information environment are able to rely on such centers for scientific excellence, but they also need to address possible conflict of interest, since usually they are also involved in clinical trials or basic research with matching funds of industry.

The mechanism design approach can be useful with that respect since it can help with implementation of rules for different types of interactions (market interactions, interactions between professionals and industry, between research centers and physicians). Moreover, the limits to address the sources of heterogeneity between practices with tools such as "Yardstick competition" [24, 25], implemented with comparative accounts between practices, may be addressed with the fast digitalization of the health system.

\section{Appendix}

\section{Appendix 1: Brief Review of Specificities of Medical Markets}

Medical markets have already been analyzed with a game theoretic approach. For instance, Libby Wang Su Sheng et als, IIE 2008, addressing the asymmetry of information at a micro level between physicians and patients with a Nash Equilibrium model

Specific concepts need to be used to analyze the medical markets, they have been introduced in the economic analysis originally by Fuchs [26] who examined several disequilibrium prices in some of the medical markets (especially the excess supply in the case of general surgery), then Evans who described induced demand in North America [27]: the literature on principal agent, especially on asymmetries of information between physicians and patients also led to explore the conditions under which the physicians were in a better position to decide for their patients; more recently an additional concept of demand induced supply was also introduced by Sina Shih et ales [28] to take into account the access to information from patients especially from internet and Direct to Consumer (DTC) information [1].

However, due to the constraints imposed by ethics principles, especially medical ethics, moral values, religious beliefs, there are additional requirements for a representative medical market using a mechanism design approach.

\section{Appendix 2: Origins of the Contributions}

A stream of research papers was published after doing research on discrete choices, using conjoint micro data, to measure physicians' preference shifts under various financial implicit restraints. In Europe, it includes an international cost sensitivity analysis on primary care physicians, to measure their sensitivity to various patient economics in six different health systems and cost sharing mechanisms. In North America, it led to two qualitative research projects: one in Boston, with physicians'focus groups on asthma patients and "thinking about cost"; one in Canada, with a qualitative research on physicians in Ontario for reform of the provincial formulary on drug budget.

The conjoint design, resulting from this type of research, called "reversed conjoint design", provides an algorithm to countervail information from the biopharmaceutical companies and other economic stakeholders. It was presented at the Society for Medical Decision Making in Toronto, and then at Ispor conference in Montreal in 2014, on physicians'choices for an application on diabetic type II. These steps provide a roadmap to integrate it in a decision support system, useful for medical or health systems, with a choice architecture including cost cognitive cues.

Recent methodological steps on this conjoint model and his application for medical systems include recent meta reviews of judgement studies comparing the field of medical sciences with business, education and psychological sciences [29], consistency issues of interstate study designs and comparisons of cost cues identifications with this psychological models versus other approaches (presented at MCDA-EWG, Trento, 2019), relevance of the axiomatic system on consistency from mathematical economists for validity of the algorithm, identification of transaction costs in primary care targeting for re-engineering of workflows between clinical and administrative tasks.

The new data elements, measured with these conjoint and micro data, represent modules of economic cues, namely similar type of information than the multi-cue cognitive medical system with clinical information; they help to detect interferences of economics (patient, physician or business economics) with clinical judgement. The importance of this psychological approach is to capture not only billing, prices or financial explicit information processed by physicians or other decision makers, but also implicit restraints that limit for instance patient adherence.

To implement this new type of matching algorithm, an engineering approach appears quite useful. This "reversed study design" incorporates economic/ cost criteria and modifies the usual context for choices among alternatives for treatments (or diagnostic and prognostic choices). The reversed mechanisms, used in the game called market design, may be used in order to design the choice architectures in a decision support system and computer codes.

The first series of papers only integrate characteristics of the 
players and interactions with providers, patients and payers. This research milestone aimed to use various layers of information including not only effective data information on market environment, but also on the psychological environment with information used to influence and persuade actors on such markets. It has been largely developed for pharmaceutical marketing. This approach uses similar type of information as counter detailing information to ensure fair comparative scientific evidence and comparative information for competitive markets. Originally initiated by two researchers from Boston, a physician Dr J Avorn and a sociologist S Soumerai, counter detailing in medicine proves to be very successful to countervail various sources of information on clinical evidence (academic detailing in medicine). The collaboration between Prof $\mathrm{C}$ Huttin and $\mathrm{J}$ Avorn lead to include not only clinical predictors but also economic characteristics. Prof Huttin continued in Europe with implicit economic information (not only prices or copay and other payment forms); these methods were especially useful to fight corruption in Southern Europe. The premises of multi cue systems for multiple groups of decision-makers has been initiated and will require strict ethical rules and proper study designs for further experiments. Using different layers of information on both sides of demand and supply, lead to identify the main actors that could use the counter detailing information (agents such as companies, doctors, pharmacists, patients and payers).

\section{Acknowledgements}

Prof Christine Huttin is grateful to Prof Sally Brailsford for the invitation to CORMSIS Seminar 2020-2021, October $9^{\text {th }} 2020$ and to UC Louvain and Vlerick Business school in Belgium for access to library services.

\section{References}

[1] Huttin CC Economics and Medical Decision Making, ed Scholars' Press, 2017.

[2] Christian B and Griffiths T, 3 "Algorithms to Live By", Picador edition, 2017.

[3] Hurwicz L "The theory of economic behavior" American Economic Review 35 (5): 909-25, 1945.

[4] Hurwicz L "The Design of Mechanisms for Resource Allocation, "Richard T Ely Lecture, American Economic Review 63 (2): 1-30, 1973.

[5] Van Neuman J and Morgenstern O, "Theory of Games and economic behaviour, Princeton, NJ, Princeton University Press, 1944, 1947, 1953.

[6] Hurwicz L, Maskin E, Myerson R lecture on the foundations of mechanism design theory, Nobel Prize, Oct 2007, Nobel Prize foundation.

[7] Harsanyi JC " Games with incomplete information played by Bayesian players, the basic model" Management Science, Nov 1967: 127-261.
[8] Roth AE “ The economics of matching: stability and incentives" Mathematics of Operational Research 7 (4): 61728, 1982.

[9] Roth AE and Wilson RB How Market Design emerged from Game Theory: a mutual interview, Journal of Economic Perspective, vol 33 (3) Summer 2019: 118-143.

[10] Roth AE, Tayfun Sonmez, Utku Unver "Kidney Exchange", Quaterly Journal of Economics 119 (2): 457-88, 2004.

[11] Villa S, Patrone F “ incentive compatibility in kidney exchange problems" Health Care Management Science, Dec 2009, Vol 12 (4): 351-362.

[12] Zhan, Yufeng; Xia, Yuanqing; Zhang, Jiang; Li, Ting; Wang, $\mathrm{Yu}$ "An incentive mechanism design for mobile crowdsensing with demand uncertainties" Information Sciences, August 2020, Vol 528: 1-16.

[13] Glaeser EL “A review essay on Alvin Roth's Who gets What - and Why?" Journal of Economic Literature 2018, Vol 55 (4): 1602-1614. AE.

[14] Huttin CC "Engineering approaches in Life Science and HealthCare, American Journal of Biomedical Science and Research, August 2019, ISSN: 2642-1747 AJBSR. MS. ID. 000838 .

[15] Nisan N, Ronen A, "Algorithmic mechanism design" Proceedings of the $31^{\text {st }}$ annual ACM symposium on theory of computing: 129-140, 1999.

[16] Su-Sheng W, Zhao-Kun, Charlene X, Jing X "Bayesian Nash Equilibrium analysis of medical market under asymmetry information" IEEE, 2008.

[17] Charles C, Gafni A, Wheelan T «Decision making in the physician-patient encounter: revisiting the shared treatment decision-making model, Soc Sci Med 1999, Sep 49 (5): 651-61.

[18] Huttin CC "Choices of Multistakeholders for economic models towards precision medicine" (Stockholm, Eupha). European Journal of Public Health, Dec suppl (S201701214), 2017.

[19] Bradlow J, Coulter A Effects of fundholding and indicative prescribing schemes on general practitioners' prescribing costs. British Medical Journal, 307 (6913): 1186-1189, 1993.

[20] Segal L; Donato R, Richardson J, Peacock S "Strengths and limitations of competitive versus non competitive models of integrated capitated fundholding" Journal of Health Service Research and Policy, 7 Suppl 1: 56-64, 2002.

[21] Croxon B, Propper C, Perkins A “ Do doctors respond to financial incentives? The UK family doctors and the GP fundholder scheme" Journal of Public Economics, 79 (2): 375 398, 2001.

[22] Chevreul K and Durand Zaleski I "Cost containment in France: assessment of current policy initiatives" Eurohealth, Vol 18 (2), 2012.

[23] Fitzegerald L, Ferlie E, Wood M, Hawkins C “ Evidence into Practice? An exploratory analysis of the interpretation of evidence " in The organizational behaviour in health care: the research agenda. Basingstoke, MacMillan, 1999.

[24] Joskow PL "Reimbursment Policy, Cost Containment, and Non Price Competition. " Journal of Health Economics, Vol 2 (1983): 167-174. 
[25] Shleifer A, "A theory of yardstick competition" Rand Journal of Economics, Vol 16, N3, Autumn 1985.

[26] Fuchs RV, Health Care and the United States Economic system, Chapter 1, in the Health Economy, Harvard University Press, 1986.

[27] Evans AR Supplier-Induced Demand: some empirical evidence and implications in the economics of Health and Medical Care, Mark Perlman London, MacMillan, 1974.

[28] Sina Shih YC, Tai Seale M Physicians' perceptions of demand-induced supply in the information age: a latent class model. Health Economics 21 (2012): 252-269.
[29] Huttin CC Clinical judgment research on economic topics: role of congruence of tasks in clinical practice. Technology and Health Care 25: 353-365, March 2017.

[30] Huttin CC "Prescribing Budget Decision Making Process in a Primary Care Group, Position of practices", Working paper series, University of Warwick, CHESS, Dec 1999 (unpublished).

[31] Glennerster $\mathrm{H}$ " The UK health reforms" in the changing roles of government and the market in HealthCare systems" edited by Chinitz DP and Cohen MA, JDC-Brookdale Institute, Israel, 1993. 